\title{
Myopic Peripapillary Pits with Spatially Corresponding Localized Visual Field Defects: A Progressive Japanese and a Cross-Sectional European Case
}

\author{
Yoshiyuki Kita $^{a}$ Gábor Hollób $^{b}$ Fumihiro Narita ${ }^{a}$ Ritsuko Kita ${ }^{a}$ \\ Akito Hirakata ${ }^{a}$ \\ aDepartment of Ophthalmology, Kyorin University School of Medicine, Tokyo, Japan; \\ bepartment of Ophthalmology, Semmelweis University, Budapest, Hungary
}

\section{Keywords}

High myopia - Optical coherence tomography angiography · Peripapillary pit · Retinal nerve fiber layer thinning · Visual field defect

\begin{abstract}
We report 2 peripapillary pit cases, in which the isolated visual field defects spatially correspond to the pit-related retinal nerve fiber layer and optical coherence tomography (OCT) angiography (OCTA) perfusion damage areas. A high myopic eye of a 39-year-old Japanese male patient, and a moderate myopic eye of a 47-years old Caucasian female patient were evaluated with OCT, OCTA, and visual field testing for peripapillary pits and spatially corresponding localized visual field defects. In the Japanese patient a temporal and in the Caucasian patient an inferotemporal peripapillary pit was confirmed, both spatially associated with a myopic peripapillary atrophy area. In both cases, the retinal nerve fibers herniated into the pit. En face OCT and OCTA revealed retinal nerve fiber bundle defects and reduced vessel density in the corresponding areas, both projecting to the pit. The visual field showed localized scotomas spatially corresponding to the nerve fiber bundle/OCTA defects in both patients. The visual field defect was a progressing (extending and deepening) paracentral scotoma in the Japanese patient, and a localized superior paracentral and superior arcuate scotoma in the Caucasian patient. Our cases show that peripapillary pits occurring in both Japanese and white European eyes can cause localized retinal nerve fiber bundle and OCTA damage and visual field defects of which some can worsen during the follow-up. To separate scotomas due to peripapillary pits and glaucoma is therefore of clinical importance and requires special attention from ophthalmologists.
\end{abstract}


Kita et al.: Peripapillary Pit and VF Defect

\section{Introduction}

In contrast to optic disc pit, which is a relatively common condition, the peripapillary pit is a rare abnormality. Definite diagnosis of a peripapillary pit requires optical coherence tomography (OCT) evaluation since the slit lamp biomicroscopy findings are not always informative and specific [1-3]. In eyes with an axial length between 29.5 and $32.8 \mathrm{~mm}$, the peripapillary pit was spatially associated with a posterior staphyloma [1]. It has been reported that thinning of the circumpapillary retinal nerve fiber layer (cpRNFL) can be progressive in eyes with a peripapillary pit [2]. However, to our knowledge no report on the spatial relationship between a peripapillary pit and a localized visual field defect has been published. In the current case report, we present a Japanese patient with a peripapillary pit and a corresponding localized progressive paracentral visual field defect, and a cross-sectional case of a white European (Caucasian) patient with a peripapillary pit and 2 spatially corresponding, localized paracentral and arcuate visual field defects.

\section{Case Report}

\section{Case 1}

A 39-year-old Japanese man visited his local eye clinic for blurred vision of his right eye. He was referred to the Kyorin University Hospital in July 2015. His best-corrected visual acuity was 1.0 on both eyes. The spherical equivalent of the refractive error was $-7.0 \mathrm{D}$ on the right (axial length: $27.24 \mathrm{~mm}$ ) and $-6.5 \mathrm{D}$ on the left eye (axial length: $26.68 \mathrm{~mm}$ ). The untreated intraocular pressure (IOP) was 17 and $15 \mathrm{~mm} \mathrm{Hg}$, respectively. Slit lamp biomicroscopy revealed a temporal retinal nerve fiber layer defect, a visible temporal peripapillary pit, and myopic type of glaucomatous optic neuropathy on the right eye (Fig. 1a). No pit or glaucomatous damage was seen on the left eye, and no posterior staphyloma was identified in either eye. Using the Humphrey field analyzer (Carl Zeiss Meditec, Dubrin, CA, USA; 10-2 Swedish interactive threshold algorithm standard program), a superior paracentral scotoma was found on the right eye (Fig. 1b). Using the Humphrey 30-2 Swedish interactive threshold algorithm standard test, an expansion of the blind spot toward the nasal side was found (Fig. 1b). The visual field of the left eye was normal. Spectral-domain OCT (Cirrus HD-OCT; Carl Zeiss Meditec, Dubrin, CA, USA) revealed a localized thinning of the inferior cpRNFL thickness and a spatially corresponding thinning of the inferior macular ganglion cell-inner plexiform layer (mGCIPL) thickness (Fig. 1e, f). The horizontal swept source OCT (DRI OCT-1 Atlantis, Topcon, Tokyo, Japan) scans showed a temporal peripapillary pit inside a myopic peripapillary atrophy and herniation of the retinal nerve fibers in the pit area (Fig. 1g). OCT angiography (OCTA; Cirrus HD-OCT) revealed reduced vessel density corresponding to the pit area (Fig. 1h). Our final diagnosis was a temporal peripapillary pit and normal-tension glaucoma with predominantly inferior structural loss of the right eye, and our opinion was that the localized paracentral scotoma was caused by the pit, and not by glaucoma. The left eye had high myopia without any glaucomatous damage. In December 2017, 17 months after the initial visit, the localized paracentral visual field defect showed progression (Fig. 1c). Latanoprost was started on the right eye. The IOP of the right eye decreased to $12 \mathrm{~mm} \mathrm{Hg}$ at 3 months after treatment initiation, and remained between 11 and $14 \mathrm{~mm} \mathrm{Hg}$ in all subsequent visits. Four years after the initial visit, the isolated paracentral scotoma showed further extension and deepening (Fig. 1d) while no other visual field defect appeared on the 30-2 visual field (Fig. 1d). The thickness values measured for cpRNFL and mGCIPL did not change in a detectable manner after the initial visit, potentially due to floor effect (Fig. 1e, f). These

\section{Karger'}


a

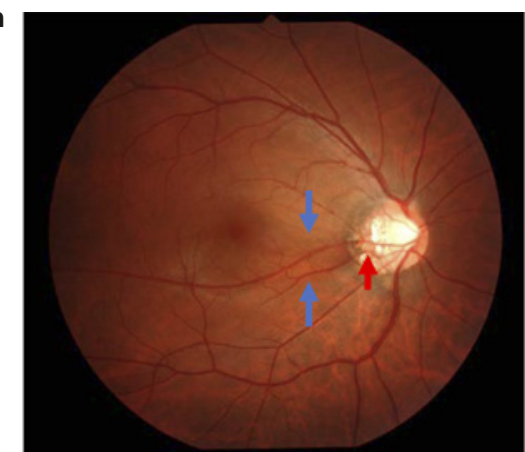

10-degree visual field 30-degree visual field $\mathbf{g}$

b

C
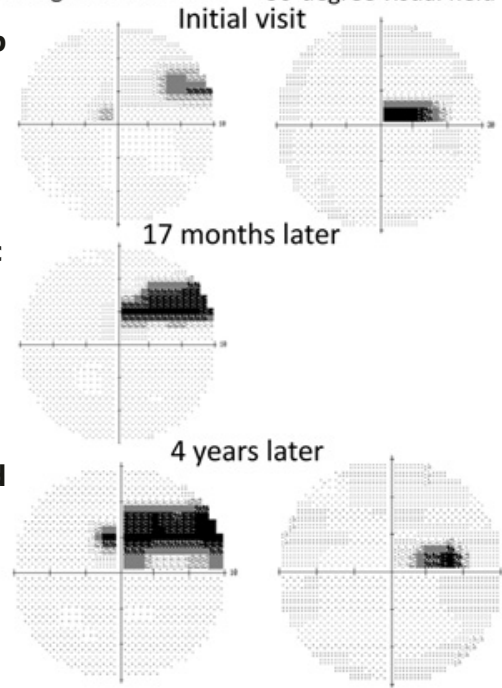

e Avera

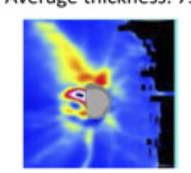

f
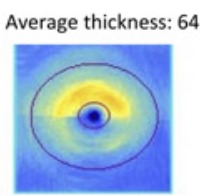

Average thickness: 74

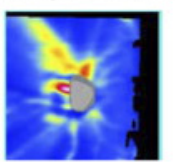

Average thickness: 62

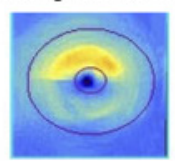

Average thickness: 78

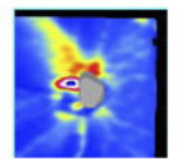

Average thickness: 63

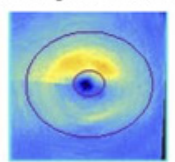

h
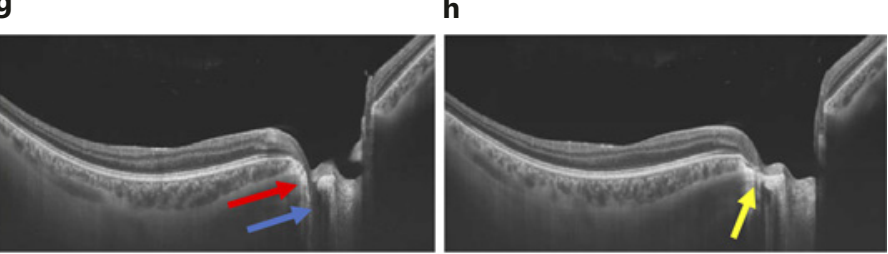

i
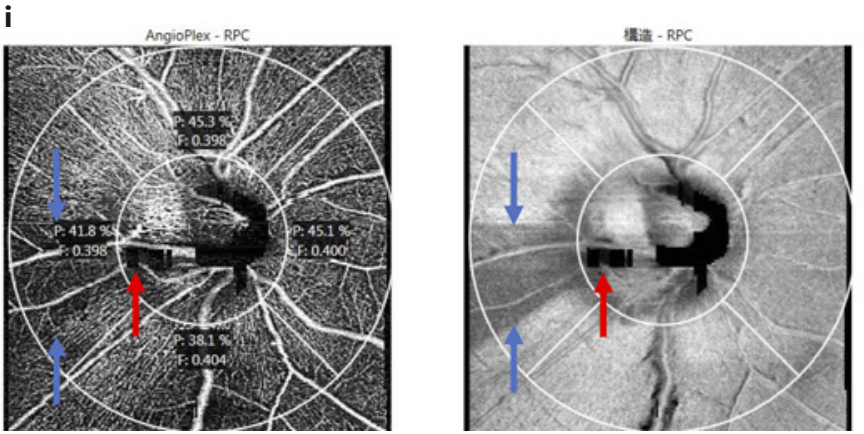

Fig. 1. Fundus photograph, Humphrey visual field, OCT and OCTA results of the right eye of the Japanese patient. a An orange circular pit in the peripapillary atrophy area (red arrow), a spatially corresponding retinal nerve fiber layer defect (blue arrows), and glaucomatous neuroretinal rim loss are present. b-d The grayscale of the central $10^{\circ}$ (left side) and $30^{\circ}$ (right side) visual field show a superior paracentral scotoma spatially corresponding to the peripapillary pit. $\mathbf{b}$ The initial-visit MD and PSD of $10^{\circ}$ visual field were -1.16 and $5.38 \mathrm{~dB}$, respectively. c At 17 months after initial visit, the corresponding MD and PSD values were -3.34 and $10.02 \mathrm{~dB}$. d At 4 years after initial visit, the MD and PSD of $10^{\circ}$ visual field were -5.21 and $11.81 \mathrm{~dB}$, respectively. $\mathbf{b}, \mathbf{d}$ The paracentral scotoma progressively extended and deepened during the follow-up. In the $30^{\circ}$ visual field, a superior paracentral scotoma was found. The initial and final MD values were -0.11 and $-0.54 \mathrm{~dB}$, respectively. However, no Bjerrum's scotoma, nasal step, and generalized depression were seen. $\mathbf{e}, \mathbf{f}$ cpRNFL thickness and mGCIPL thickness measured with the Cirrus HD-OCT. Left: initial visit, middle: 45 months after the initial visit, and right: 4 years after the initial visit. No change is measured. $\mathbf{g}$ Topcon Swept source OCT image of the peripapillary pit (red arrow). The pit is located in the temporal myopic peripapillary atrophy area, and contains a cleft that crosses the sclera (blue arrow). Herniation of the retinal nerve fibers toward the pit is visible. $\mathbf{h}$ Topcon swept source OCT image of the optic disc in a slice slightly above the level shown in (g). The yellow arrow indicates the temporal myopic peripapillary atrophy area (gamma zone) and shows that the pit does not involve the optic disc. $\mathbf{i}$ OCTA image and the corresponding en face structural image of the cpRNFL (Cirrus HD-OCT). The wedge-shaped retinal nerve fiber defect and the spatially corresponding wedgeshaped peripapillary vessel density reduction are indicated with blue arrows. The red arrow indicates the peripapillary pit and the related lack of perfusion. OCT, optical coherence tomography; OCTA, optical coherence tomography angiography; MD, mean deviation; PSD, pattern standard deviation; cpRNFL, circumpapillary retinal nerve fiber layer; mGCIPL, macular ganglion cell-inner plexiform layer. 
findings suggested that the visual field progression was not related to normal-tension glaucoma which remained stable and controlled during the 4-year follow-up.

Case 2

A 47-year-old systemically healthy white European (Caucasian) female asked for a second opinion in the Glaucoma Unit of the Semmelweis University, Budapest, in January 2020. She was treated for open-angle glaucoma with a prostaglandin analog on both eyes for $<1$ year. Funduscopy revealed a tilted myopic disc and an extensive temporal peripapillary atrophy with a suspected inferotemporal peripapillary pit on the right eye. Using the AngioVue OCT (Optovue Inc., Fremont, CA, USA), the inferotemporal peripapillary pit inside a myopic peripapillary atrophy was confirmed (Fig. 2a, b). The retinal nerve fibers were herniated in the pit area, and the pit was associated with a localized intrachoroidal cavitation (ICC; Fig. 2b). The left eye had a normal optic nerve head, normal cpRNFL pattern and thickness, and a normal visual field (data are not shown). Her best corrected visual acuity was 1.0 on both eyes with a refractive error of $-5.5 \mathrm{D}$ on the right and $-5.0 \mathrm{D}$ on the left eye, and the central cornea thickness was 554 and $542 \mu \mathrm{m}$, respectively. After a 1-month medication washout, the daytime phasing IOP was stable: $19 \mathrm{~mm} \mathrm{Hg}$ on the right and $17 \mathrm{~mm} \mathrm{Hg}$ on the left eye. The corresponding en face structural and OCTA images in the radial peripapillary capillaries layer
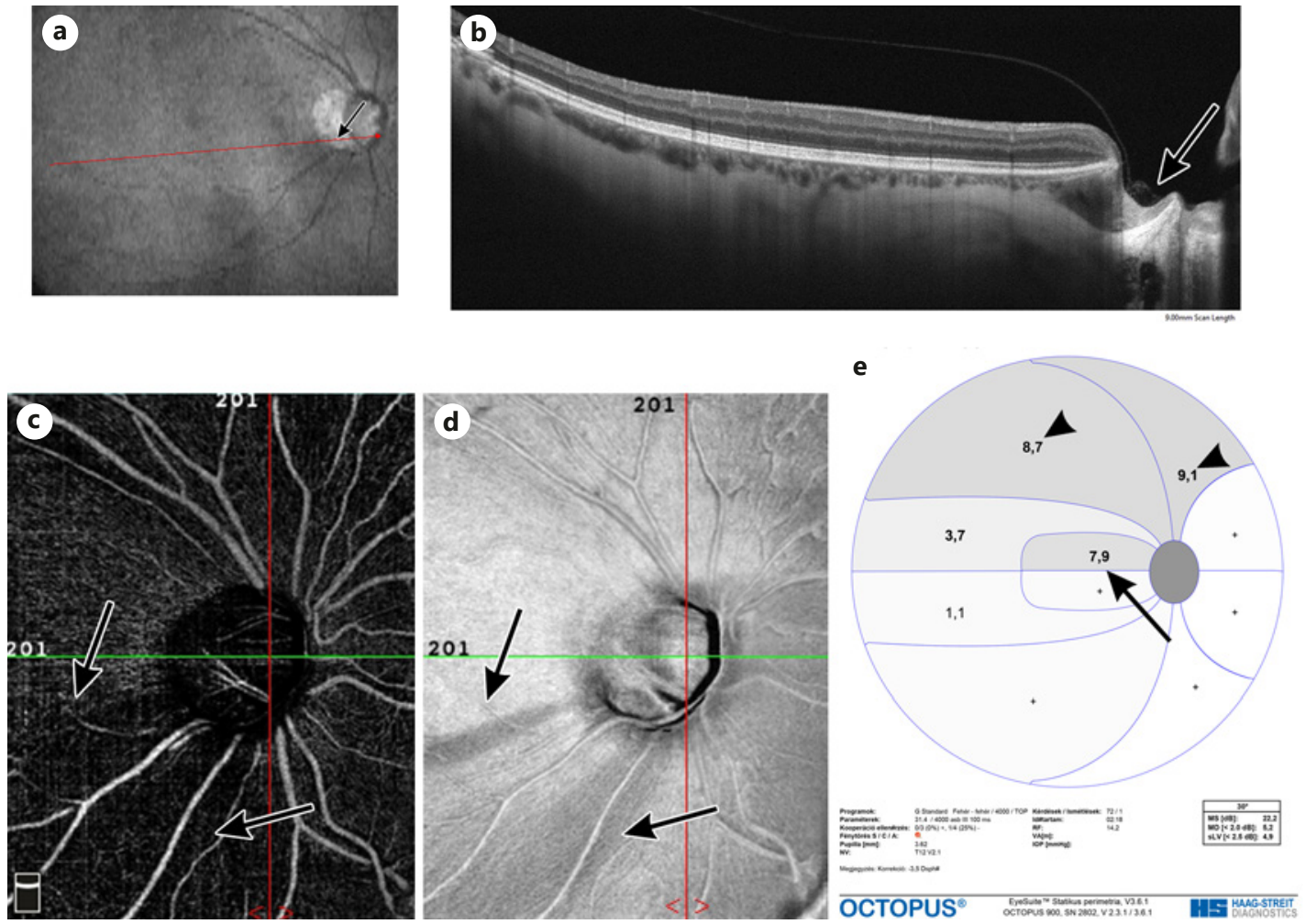

Fig. 2. OCT and OCTA images and Octopus perimetry visual field test results of the right eye of the white European (Caucasian) patient. a The red OCT scanning line crosses the temporal peripapillary atrophy area where the peripapillary pit (arrow) takes place. $\mathbf{b}$ The peripapillary pit is indicated with an arrow on the OCT scan. On the en face OCTA (c) and retinal nerve fiber layer (d) scans, the spatially corresponding perfusion (vessel density) reductions and retinal nerve fiber bundle defects are indicated with arrows. e The superior paracentral visual field cluster defect (arrow) corresponds with the inferior temporal retinal nerve fiber bundle defect. The superior arcuate visual field defect, which involves 2 visual field clusters (arrow heads), corresponds with the wide inferotemporal retinal nerve fiber bundle defect. OCT, optical coherence tomography; OCTA, optical coherence tomography angiography. 
(Fig. 2c, d) showed inferotemporal RNFL bundle defects and spatially corresponding reductions of capillary perfusion. The central $30^{\circ}$ visual field was investigated with Octopus perimetry (TOP strategy, corrected cluster analysis). Figure 2e shows a localized superior paracentral visual field defect (cluster mean defect: $7.9 \mathrm{~dB}$ ) which spatially corresponds with the inferior temporal RNFL and capillary perfusion defects, and a superior paracentral visual field defect involving 2 clusters (cluster mean defects: 8.7 and $9.1 \mathrm{~dB}$, respectively) which spatially corresponds with the wide inferotemporal RNFL and capillary perfusion defects. Figure $2 \mathrm{a}$ and $\mathrm{b}$ show that both RNFL bundle defects project to the pit area; thus, they are related to the peripapillary pit. The patient is currently under observation with no IOP lowering treatment.

\section{Discussion and Conclusions}

Peripapillary pits are not uncommon abnormalities in pathological myopia, but the presence of a peripapillary pit that is visible with funduscopy is rare. Ohno-Matsui et al. [1] reviewed the medical records of 198 pathological myopia patients whose papillary and peripapillary regions were examined with swept source OCT. Twenty-two out of the 198 patients $(11.1 \%)$ were found to have a peripapillary pit. Of the 22 cases, a clinically visible peripapillary pit was observed in only 2 eyes [1]. Others also reported 2 cases of visible peripapillary pit $[2,3]$; thus, only 4 cases were published. Of note, all previously reported peripapillary pit cases occurred in East Asian (Japanese [1], Chinese [3], and Korean [2]) eyes. Therefore, our second case is the first which indicates that peripapillary pits can also occur in white Europeans.

While our 2 cases share common features (a pit inside a temporal $180^{\circ}$ peripapillary atrophy area, herniation of the retinal nerve fibers into the pit area, and presence of spatially corresponding localized scotomas in the visual field), they also show differences. In case 1 , the pit is associated with a dehiscence of the sclera, which is not seen in case 2. However, in case 2, a localized ICC is attached to the pit, which is not seen in case 1. ICC with a corresponding defect of the retina has been described earlier as an abnormality which can be associated to peripapillary pits [4-6]. Case 2 fits to the previously described cases. Our cases suggest that peripapillary pits may be divided into different categories based on their structural characteristics even when the peripapillary pit-related visual field damages are similar.

In the previous publications, the relationship between the peripapillary pits and the potentially related visual field deteriorations remains unknown. However, in our 2 cases, we demonstrate a clear spatial relationship between the location of the retinal nerve fiber layer/ perfusion damage related to the peripapillary pit and a localized scotoma in the superior paracentral visual field (both cases) and in the superior clusters of the $30^{\circ}$ visual field (case 2 ). In addition, in case 1 , a progressive extension and deepening of the isolated superior paracentral scotoma was documented during the 4-year follow-up, without the development of any other (glaucomatous) visual field alteration. In this case, the standard OCT parameters (cpRNFL thickness and mGCIPL thickness) approved for the detection and quantification of glaucoma progression remained stable, and the IOP was well controlled on medication. These findings suggest that in case 1 , the progression of the isolated scotoma was related to the peripapillary pit. It has been reported that the size of the disc and the myopic conus is greater in eyes with a pit compared to eyes without a pit, which suggests that mechanical expansion of the papillary region is the primary cause of the development of peripapillary pits in highly myopic eyes [1]. One may speculate that parallel to the increase of the axial length, the peripapillary area experiences mechanical stress, which may play a role in the development and progression of peripapillary pits and the related visual field alterations.

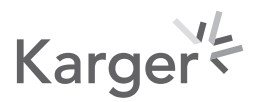


Kita et al.: Peripapillary Pit and VF Defect

Our cases indicate that myopic peripapillary pits can occur in Caucasian (white European) patients and can correspond with localized scotomas in the paracentral and arcuate visual field regions, and the peripapillary pit-related isolated visual field deterioration can progress over time, suggesting progression of the pit itself. Further long-term investigations are needed to clarify if our current findings are generally valid for peripapillary pits in myopic eyes.

\section{Statement of Ethics}

The patients have given a written informed consent for the publication of this report.

\section{Conflict of Interest Statement}

Gábor Holló is a consultant of Optovue Inc and Zeiss. The other authors declare no conflict of interest.

\section{Funding Sources}

This study was not funded.

\section{Author Contributions}

All authors collected and interpreted the patient's clinical findings. Y.K. and G.H. contributed to the writing and editing of the manuscript. All authors reviewed the literature. All authors read and approved the final version of the manuscript.

\section{References}

1 Ohno-Matsui K, Akiba M, Moriyama M, Shimada N, Ishibashi T, Tokoro T, et al. Acquired optic nerve and peripapillary pits in pathologic myopia. Ophthalmology. 2012;119(8):1685-92.

2 Lee EJ, Kim TW. Progressive retinal nerve fiber layer atrophy associated with enlarging peripapillary pit. J Glaucoma. 2017;26(2):e79-81.

3 Hu X, Dai Y, Jonas J, Sun X. Peripapillary gamma zone pit as dehiscence between Elschnig's border tissue and Bruch's membrane with herniation and defect of the retinal nerve fiber layer. BMC Ophthalmol. 2016;16(1): 143.

4 Ohno-Matsui K, Shimada N, Akiba M, Moriyama M, Ishibashi T, Tokoro T. Characteristics of intrachoroidal cavitation located temporal to optic disc in highly myopic eyes. Eye. 2013;27(5):630-8.

5 Chen Y, Ma X, Hua R. Multi-modality imaging findings of huge intrachoroidal cavitation and myopic peripapillary sinkhole. BMC Ophthalmol. 2018;18(1):24.

6 Kita Y, Inoue M, Holló G, Kita R, Sano M, Hirakata A. Preserved retinal sensitivity in spatial correspondence to an intrachoroidal cavitation area with full thickness retinal defect: a case report. BMC Ophthalmol. 2016; 16(1):186.

\section{Karger'k}

